\title{
Siglas, acrónimos y abreviaturas en la prensa especializada económica en alemán y español
}

Initialisms, acronyms and abbreviations

in specialized texts of Spanish and

German economic written media

\section{Ana Medina Reguera}

Universidad Pablo de Olavide

\section{Alice Stender}

Universidad Pablo de Olavide

Tendencias actuales en

traducción

para el

ámbito de la

economía y

los negocios

2019
ONOMÁZEIN

Número especial $\vee$ - Tendencias actuales en traducción para el ámbito de la economía y los negocios: 94-113 DOI: 10.7764/onomazein.tradecneg.05 ISSN: 0718-5758

\section{(c) $\bigodot_{\mathrm{BY}} \bigodot_{\mathrm{ND}}$}

Ana Medina Reguera: Universidad Pablo de Olavide, Departamento de Filología y Traducción.

| E-mail: ammedreg@upo.es

Alice Stender: Universidad Pablo de Olavide, Departamento de Filología y Traducción.

| E-mail: aste@upo.es

Fecha de recepción: julio de 2018

Fecha de aceptación: diciembre de 2018 


\section{Resumen}

El objetivo principal de este trabajo es analizar contrastivamente las siglas, acrónimos y abreviaturas del lenguaje especializado económico en las lenguas española y alemana, con la ayuda de un corpus comparable de noticias económicas procedentes de la prensa especializada de España y Alemania. La crisis económica de 2007 es la base temática para este estudio de corpus, cuyos textos abarcan hasta 2015. Las siglas son un recurso característico del lenguaje económico y son útiles para optimizar el lenguaje mediante la reducción de términos financieros, nombres de instituciones, etc., mucho más largos en su forma desarrollada. No obstante, frecuentemente, en lugar de ayudar a la comprensión conceptual, ocurre lo contrario, pues el mensaje queda codificado y la comprensión se obstaculiza. Se observa una relación entre la economía del lenguaje (que impulsa el acortamiento) y la densidad terminológica propia del lenguaje de la economía (a mayor número de siglas, mayor densidad del texto especializado). El estudio también concluye que los conceptos de abreviatura, sigla y acrónimo se definen de manera bastante más clara para el español que para el alemán, donde no existe un consenso unánime sobre la clasificación y denominación de palabras abreviadas. Por último, se abordan las estrategias de traducción posibles y se clasifican semánticamente las formas encontradas en el corpus.

Palabras clave: abreviaturas; siglas; acrónimos; lenguaje económico; traducción económica.

\section{Abstract}

The main aim of this work is to perform a contrastive analysis on the acronyms and abbreviations of the language of economics in Spanish and German, with the aid of a comparable corpus of economic news published in the specialised press in both countries. The 2007 economic crisis is the thematic basis of this corpus study, whose texts encompass the period up until 2015. Acronyms are a characteristic resource of the language of economics and serve to streamline it by reducing the length of words, the names of institutions, etc., which are much longer in their normal format. However, instead of contributing to conceptual understanding, the opposite often occurs, since the message is coded and, therefore, impedes this. It is possible to observe a relationship between the language of economy (which encourages this shortening) and its terminological density (the greater the number of acronyms, the greater 
this density in specialised texts). The study also concludes that the concepts of abbreviation and acronym are more clearly defined in Spanish than in German, in which there is no unanimous consensus on the classification and designation of abbreviated words. Lastly, possible translation strategies are addressed and corpus examples semantically classified.

Keywords: acronyms; abbreviations; economic language; economic translation; German. 


\section{Introducción}

El lenguaje económico cuenta con distintos elementos léxico-semánticos que lo definen como lenguaje especializado, entre los que destacan, por ejemplo, el uso de metáforas de diversos dominios y la alta frecuencia de préstamos de otras lenguas ${ }^{1}$. Cada una de estas características tiene una finalidad en el discurso: mientras que las metáforas tienen como objetivo ayudar a visualizar y, consecuentemente, a comprender conceptos abstractos, los préstamos, calcos y extranjerismos dan una forma lingüística a conceptos o productos nuevos. En el caso de las formas prestadas, a diferencia de lo que ocurre con las metáforas, la comprensión puede verse obstaculizada, pues el empleo de neologismos procedentes de otras lenguas contribuye a que el texto pueda convertirse en un código de difícil comprensión para los no expertos. Este trabajo versa sobre un tercer elemento clave para el lenguaje económico: las palabras abreviadas o abreviaciones. Estas pueden ser formas monoléxicas o pluriléxicas que se han sometido a un proceso de truncación lingüística y constituyen, en su conjunto, una de las características más representativas del lenguaje económico. A priori, se manifiestan como un recurso muy productivo por la inherente reducción y, por tanto, son útiles para la economía lingüística, al incrementarse la rapidez y la facilidad en la transmisión de las informaciones (Nueva Gramática de la RAE, 2009: 36; Gómez de Enterría, 1992a: 273).

Sin embargo, el uso - y, sobre todo, el abuso — de siglas y abreviaturas también dificulta la comprensión. En palabras de Corbacho (2006: 19):

La concisión y la capacidad de transmitir un contenido conceptual con un material lingüístico mínimo estimula a los profesionales a emplear las siglas, aunque su uso en círculos no especializados tiende a complicar el entendimiento de las mismas por su carácter notoriamente críptico.

También se señala en la Nueva Gramática que su uso requiere un mayor esfuerzo de atención y de memoria en la lectura.

En general podemos hablar de tres tipos de abreviaciones en el discurso económico: siglas, acrónimos y abreviaturas. Las primeras se definen, según la última edición del DRAE, como "abreviación gráfica formada por el conjunto de letras iniciales de una expresión compleja”. El término sigla se aplica tanto a la secuencia completa como a cada una de las iniciales que la integran, y una vez constituidas, "pueden comportarse como cualquier palabra y admitir la formación de derivados, aunque no todos pasen a los diccionarios: peneuvista ('relativo al PNV', Partido Nacionalista Vasco), otanización (de OTAN)” (RAE, 2009: 32).

1 Para una caracterización general del lenguaje económico véase Mateo Martínez (2007), Suau Jiménez (2010), Pizarro Sánchez (2011) o Gallego-Hernández (2012). Específicamente para la lengua alemana, Hundt y Biadala (2015), Spillner (1992) o Moss (2009). Estudios contrastivos alemán-español destacan Corbacho (2003, 2007), Medina Reguera y Stender (2012, 2013) y Stender (2016). 
Los acrónimos, por su parte, son siglas pronunciables como palabras que se integran en la lengua como sustantivos y se pronuncian como tales. En ocasiones el lector entiende el significado de la sigla sin que sea especialmente relevante cada uno de los componentes que la forman: láser, que procede de la expresión inglesa l(ight) a(mplification) (by) s(timulated) e(mission) (of) r(adiation), o radar, también del inglés ra(dio) d(etecting) a(nd) r(anking) (RAE, 2009: 37). Como puede verse en el último ejemplo, la unión no resulta siempre de unir la primera letra de cada palabra, siendo posibles otras muchas combinaciones: Mercosur (Mercado Común del Sur), Eurasia (Europa y Asia). El significado sí emana siempre de la combinación de las palabras que lo constituyen (así, apartotel o apartahotel es la suma de apartamento y hotel). Cuando se añaden o quitan fonemas para ayudar a la pronunciación, algunos autores hablan de "sigloides" en lugar de siglas (Gómez de Enterría, 1992b), aunque la Nueva Gramática considera siglas a todas estas variantes (vid. RAE, 2009: 168).

Una abreviatura, por último, es, según el DRAE, una

representación gráfica reducida de una palabra o de un grupo de palabras, obtenida mediante un procedimiento de abreviación en que se suprimen letras finales o centrales, cerrada generalmente con punto y raramente con barra.

Es un proceso propio de la lengua escrita, ya que en la oral se debe reproducir la secuencia fónica completa. Morfológicamente abundan las formas por apócope, como cap. (capítulo), cód. (código) o mín. (mínimo), y por síncopa, consistente en la pérdida de grafemas en el interior de la palabra, como en dpto. (departamento), Sra. (señora) o Dr. (doctor). No deben confundirse con siglas algunas abreviaturas que están constituidas por las iniciales de las palabras que las integran, como S. A. (sociedad anónima) o C. P. (código postal), que por su consideración como abreviaturas están separadas por puntos.

Para el alemán, el estado de la cuestión es más opaco, pues no existe un consenso unánime sobre las categorías de palabras abreviadas ni tampoco sobre la denominación general, sobre la que existen diferentes opiniones. Corbacho (2007), por ejemplo, establece Abkürzungswort para acrónimo, mientras que para Rothstein (2010) son Lautinitialwörter. Michel (2006: 70), basándose en definiciones e investigaciones anteriores, opina que

[d]ie daraus resultierenden Kurzwortklassifikationen sind zu abstrakt, um mehr zu ermöglichen, als eine Grobcharakterisierung einzelner Kurzwortbelege und eine allgemeine Zuordnung zu bestimmten Klassen.

Una de las principales dificultades para la clasificación de las palabras abreviadas del alemán consiste en la capacidad de abreviar en una sola letra cada miembro de una palabra compuesta, algo que no ocurre en español (imaginemos que, para el español, de autogestión se obtuviera $A G$ ). 
Por cuestiones de espacio, no incidiremos aquí en una descripción gramatical detallada, y citaremos únicamente las formas más representativas con sus correspondientes ejemplos, basándonos en Rothstein (2010):

- Buchstabierwort: palabras en las que se ha acortado la forma desarrollada a unas pocas letras y que se pronuncian deletreando cada una de ellas; por ejemplo, LKW(Lastkraftwagen).

- Lautinitialwort: la forma desarrollada se acorta hasta dos o más sonidos o letras que se pronuncian siguiendo las normas fonológicas como una palabra (TüV de Technischer Überwachungsverein).

- Silbeninitialwort: se acorta por sílabas, y en palabras compuestas se suele emplear la primera sílaba de cada miembro del compuesto (Kripo de Kriminalpolizei).

- Misch-Kurzform: constituye una mezcla entre las tres formas anteriores (Gema, Gesellschaft für musikalische Aufführungs- und Vervielfältigungsrechte).

Las siglas sirven para economizar el espacio y el tiempo de lectura, rasgo que es muy común en las convenciones de los lenguajes especializados. Guilbert se lamentaba en 1975 sobre las consecuencias negativas para el estilo: las denominaciones de conceptos nuevos son tan largas que se hace necesario acortarlas como sea para evitar las constantes reiteraciones a las que darían lugar (Guilbert, 1975). El origen de las siglas está directamente relacionado con los lenguajes especializados (Gómez de Enterría, 1992b), ya que en los ámbitos especializados es donde más surgen las nuevas denominaciones. Con ellas aumenta la eficacia y precisión características de estos lenguajes, cuyas formas se comportan morfosintácticamente como sustantivos comunes o propios. En cuanto a la creación del plural, se puede formalizar "en aquellas siglas que se lexicalizan plenamente y funcionan con la misma autonomía que cualquier lexema ordinario en la lengua” (Gómez de Enterría, 1992b: 270).

\section{Objetivos y metodología}

En las siguientes páginas nos centraremos en las formas abreviadas de la prensa especializada y, concretamente, en un corpus de 500 textos (260 046 palabras) comparables en alemán y en español (250 en cada lengua, con 123572 y 136474 palabras respectivamente). La temática de los textos que componen el corpus es la crisis económico-financiera y el lapso de tiempo de recopilación se ubica precisamente en los años de su duración (2007-2015). Los textos proceden de las publicaciones Handelsblatt (HD) para el alemán y Expansión (EX) y Cinco Días (CD) para el español. Para la explotación del corpus hemos utilizado la herramienta de análisis Sketch Engine, y hemos creado una wordlist en ambos subcorpus. Tras vaciar el listado manualmente, clasificamos 108 abreviaciones diferentes (types) en el subcorpus alemán (2108 tokens en total) y 113 en el español (1442 tokens). Es un porcentaje alto, si bien no hemos comparado la cifra con un corpus de referencia, al considerar que no es un dato que se necesite contrastar 
científicamente. Posteriormente, hemos analizado la lista manualmente para clasificar los distintos grupos en las siguientes categorías semánticas: a) organismos e instituciones (incluidos los partidos políticos), b) fondos e índices bursátiles, c) bancos y nombres comerciales, d) operaciones y productos financieros. A la vista de la extracción y de sus contextos en ambas lenguas, hemos dividido el análisis en cuatro aspectos: uso de formas abreviadas en titulares, traducción, ortografía y morfología, y diferencias en el uso de abreviaturas simbólicas.

\section{Análisis y resultados}

\subsection{Uso de formas abreviadas en titulares}

Las siglas y acrónimos no solo aparecen en el cuerpo del texto, donde existe también la posibilidad de desarrollar la forma la primera vez que aparecen, sino que están presentes en los titulares. El uso de las siglas en los titulares presupone conocimientos previos del receptor del texto. A continuación mostramos algunos de los numerosos ejemplos del corpus²:

\section{TABLA 1}

Siglas en los titulares de HB, CD y EX

\begin{tabular}{|c|c|}
\hline ALEMÁN & ESPAÑOL \\
\hline $\begin{array}{l}\text { EZB und Fed pumpen erneut Milliarden in den } \\
\text { Markt (A_07) }\end{array}$ & $\begin{array}{l}\text { - BNP reaviva los temores crediticios al verse } \\
\text { forzado a suspender tres de sus fondos (B_05) }\end{array}$ \\
\hline - Zukunftssorgen dominieren die Fed (A_16) & $\begin{array}{l}\text { - La Fed reduce la previsión de crecimiento de } \\
\text { EEUU y manda el dólar a mínimos (B_16) }\end{array}$ \\
\hline - JP Morgan hievt US-Börsen ins Plus (A_52) & $\begin{array}{l}\text { - JPMorgan enciende las alarmas en Wall Street } \\
\text { (B_18) }\end{array}$ \\
\hline - UBS versinkt im Subprime-Strudel (A_25) & $\begin{array}{l}\text { - UBS registra las mayores pérdidas trimestra- } \\
\text { les de la historia bancaria (B_25) }\end{array}$ \\
\hline - Dax: Es geht weiter bergauf (A_53) & $\begin{array}{l}\text { - El Ibex se 'acicala' para recibir las cuentas de } \\
\text { JPMorgan (B_53) }\end{array}$ \\
\hline $\begin{array}{l}\text { • „CoCo“-Bonds werden für Banken zum teuren } \\
\text { Vergnügen (A_182) }\end{array}$ & $\begin{array}{l}\text { - La nueva emisión de Santander muestra las } \\
\text { debilidades del mercado de CoCos (B_182) }\end{array}$ \\
\hline $\begin{array}{l}\text { - S\&P stuft Zypern hoch, Russland behält Note } \\
\text { (A_195) }\end{array}$ & $\begin{array}{l}\text { - S\&P salva a Rusia de caer en bono basura } \\
\text { (B_182) }\end{array}$ \\
\hline
\end{tabular}

2 Entre paréntesis se indican las referencias de los textos. A_número en el caso del subcorpus alemán y B_número en el subcorpus español. Recuérdese que los ejemplos del corpus comparable no son traducciones. 
Como puede verse en los ejemplos, las siglas corresponden a nombres propios, normalmente referidos a instituciones financieras y organismos internacionales:

- Países: US/EEUU

- Bancos: EZB (Europäische Zentralbank), BNP (Banco Nacional de París), Fed (Federal Reserve), UBS (Union des Banques Suisses)

- Bolsa: Dax (Deutscher Aktienindex)

- Agencia de rating (calificaciones): S\&P (Standard \& Poors)

Desde un punto de vista contrastivo, los titulares en español son más largos y los sustantivos suelen ir precedidos por sus artículos; los alemanes son más resumidos, quizás aportan menos información, raramente tienen más de un verbo u oraciones coordinadas. Ambas lenguas coinciden en el uso de metáforas (Stender, 2016) y una forma similar de uso de las formas abreviadas, en cantidad e importancia.

\subsection{Consideraciones sobre la traducción de siglas}

Las siglas constituyen una dificultad en la traducción de textos económicos, poco abordada en la bibliografía. Según Gómez Moreno (1995) o Pérez Berenguel (2003), la norma para la adaptación de una forma abreviada proveniente de un código extranjero es la traducción de cada uno de sus elementos y la posterior reordenación de sus siglas. Así se crean siglas y acrónimos que nacen como traducciones de una lengua origen a una lengua meta, como BEI (Banco Europeo de Inversiones) en vez de EIB (European Investment Bank) o FEEF (Fondo Europeo de Estabilidad Financiera) para European Financial Stability Facility (EFSF). Encontramos estos modelos de traducciones en ambos subcorpus. Por ejemplo, para el alemán EZB (Europäische Zentralbank) por ECB (European Central Bank) o IWF (Internationaler Währungsfonds) frente a IMF (International Monetary Fund). Para nuestras dos lenguas de trabajo, lo más frecuente en los textos sobre macroeconomía es que estas siglas y acrónimos provengan del inglés. Si son instituciones importantes y mantenidas en el tiempo, tanto el español como el alemán habrán acuñado su propia sigla; por ejemplo, FMI (Fondo Monetario Internacional), por continuar con el ejemplo anterior.

Pero puede ocurrir que no dispongamos de la forma desarrollada de la sigla o acrónimo en el texto original. A este respecto, Gallego-Hernández (2012: 98) afirma:

En cuanto a su traducción, autores como Elias (1997: 240-245) consideran que esta operación implica tres etapas básicas: identificación de la sigla [...]; descodificación, que supone la reconstitución del sintagma, y reexpresión. Respecto de la segunda etapa, algunos autores subrayan que el traductor puede llegar a sentirse "mortificado" por no poder descifrar en ocasiones su 
significado [...], si bien a día de hoy las posibilidades de documentación ayudan considerablemente a salvar este escollo.

En efecto, es una dificultad de traducción en textos muy especializados o cuando el traductor no está familiarizado con conceptos de economía. Al desconocimiento de la sigla puede unirse la falta de entendimiento del concepto aún una vez localizada la forma desarrollada, sobre todo en el caso de productos y operaciones financieras en los que se presupone conocimiento experto implícito. En el siguiente ejemplo, se combinan dos siglas —una de ellas alfanumérica- con lenguaje especializado (Staatshilfe, tilgen, Märkten) y metafórico (Sommerruhe, in die Schublade).

Die Wiesbadener Aareal Bank hatte mit einem AT1-Papier eigentlich noch im ersten Halbjahr ihre restlichen Staatshilfen von 300 Millionen Euro tilgen wollen. Ende Juli, als an den Märkten längst Sommerruhe eingekehrt war, legte das Institut seine „CoCo“-Pläne dann zurück in die Schublade.

Las dos siglas son anglicismos y se utilizan en el texto como sinónimos. El sintagma para la primera (ATI) es Additional Tier 1, mientras que la segunda (CoCo) se desarrolla Contingent Convertible Bonds. Para traducir el ejemplo anterior al español, tendríamos que decidir si mantener el anglicismo o traducirlo al español. Una búsqueda documental nos arroja numerosos resultados de la traducción de CoCo por Bonos Contingentes Convertibles, mientras que la forma ATI suele permanecer tal cual en textos en español. Obsérvese la explicación del anglicismo por parte del periodista en un texto de Expansión:

Basilea III permite a las entidades sumar un máximo del 1,5\% a su capital Tier 1 en forma de estos bonos que computan como Tier 1 Adicional (Additional Tier 1, en inglés, de ahí que sean conocidos por las siglas AT1) para cumplir las exigencias de solvencia que entrarán en vigor el 1 de enero de 2019.

Es decir, una vez encontrada la forma desarrollada de la sigla, y si esta no tiene una traducción fijada en español, podemos traducir el sintagma palabra por palabra, de forma más literal o bien con un equivalente dinámico en la cultura meta dependiendo del tipo de sigla: una institución propia de un país, un producto financiero, un tipo de impuesto con equivalente en la lengua meta...

Además de la traducción del sintagma original, el traductor puede optar por añadir en el texto meta la forma desarrollada en la lengua origen (es decir, sigla en inglés y forma desarroIlada en inglés) o solo la sigla, para facilitar la comprensión en el texto meta. En estos casos es frecuente que el traductor añada al paréntesis "por sus siglas en inglés":

La entidad ha aceptado pagar una multa de 205 millones de dólares como parte del acuerdo alcanzado con el Departamento de Servicios Financieros del Estado de Nueva York (DFS, por sus siglas en inglés) por violar las leyes bancarias neoyorquinas. 
En el ejemplo anterior no se desarrolla la forma en lengua original, quizás por motivos de espacio. En cualquier caso, la forma desarrollada, tanto en el inglés original como traducida a la lengua meta, puede mejorar la comprensión del receptor.

También puede ocurrir que el traductor desconozca la existencia de un equivalente acuñado en la lengua meta. Son frecuentes los productos financieros u operaciones bursátiles a los que la DGT de la UE ha acuñado un equivalente por su uso frecuente (y lo deja registrado en IATE o en artículos de la revista Punto y Coma), aunque estos términos requieren un proceso de documentación más intensivo que las siglas referidas a instituciones. Un buen uso de la web como corpus de consulta (Gallego-Hernández, 2017) es la metodología más habitual y probablemente la más ágil, si bien, además de la DGT, Fundéu, Gallardo y Gómez de Enterría (2009), el Wirtschaftslexikon de Springer Gabler (2013) o el Duden Fremdwörterbuch (2015) son otras fuentes donde es posible consultar términos económicos recientes.

Por último, el traductor puede dudar sobre si dejar la sigla en inglés, siendo el original un texto en español o alemán. A pesar de que los anglicismos están presentes tanto en el lenguaje económico español como en el alemán, ambas lenguas no se comportan igual. Los anglicismos son más tolerados en la lengua alemana que en la española, y son también superiores en número en nuestro corpus. Es necesario, por tanto, buscar siempre posibles equivalentes en la lengua meta española.

En lo referente al orden entre la sigla y su desarrollo, en el ejemplo anterior, la sigla ha sido desarrollada antes de su aparición siglada en el texto. Sin embargo, abundan ejemplos en el corpus en los que el desarrollo es posterior; por ejemplo, DEA (Drug Enforcement Administration, Administración para el Control de Drogas). En casos de instituciones propias de único país, si se opta por traducir la sigla, se procura una traducción literal evitando una diferencia innecesaria entre lenguas, y, en ningún caso, se aporta por iniciativa propia una sigla desde una forma desarrollada traducida, es decir, no convertiremos la DEA en la *ACD.

En alemán, puede ocurrir que se mantengan las siglas originales del inglés y la traducción, aunque conocida y frecuente, no se haya lexicalizado como sigla; por ejemplo:

- Inglés: United Nations Organization (UNO), Organization for Economic Cooperation and Development (OECD)

- Español: Organización de las Naciones Unidas (ONU), Organización para la Cooperación y Desarrollo Económicos (OCDE)

- Alemán: Vereinte Nationen (UNO, no *VN), Organisation für wirtschaftliche Zusammenarbeit und Entwicklung (OECD, no *OWZE)

Por otra parte, para el traductor puede ser útil buscar los términos originales que han formado los acrónimos. En muchos casos, estos han penetrado en la lengua de tal forma 
que ya no existe conciencia de que sean acrónimos. Por ejemplo, Euribor procede de la forma Eur(opean) I(nterbank) O(ffered) R(ate). Como estrategia de comprensión para la traducción puede resultar útil, así como si necesitamos usar sinónimos o explicitar una traducción del alemán al español, pues el primero admite más repeticiones de un mismo término que el segundo.

En definitiva, el criterio para desarrollar la sigla en el texto meta será el grado de conocimiento del lector tipo para nuestro texto original. Así, no desarrollaremos ONU, FMI o UE (excepto si traducimos para un público escolar o poco culto). En caso de duda, el traductor decidirá individualmente siguiendo las pautas del encargo de traducción, en función del lector meta y de la mayor comprensión posible.

\subsection{Cuestiones ortográficas y morfológicas de palabras abreviadas}

La lengua española permite más procesos de derivación que el alemán en el uso de las siglas; por ejemplo, de PNV, peneuvista, o de PP, pepero. Estas formas no pueden traducirse al alemán con una fórmula de morfología derivativa (PNV-ler*) dado que no son gramaticalmente aceptables en esta lengua. De CDU no se deriva CDU-ler*, sino CDU-Vertreter/ Wähler/Politiker, etc.

Otra diferencia se da en la elección del género. En español las siglas toman el artículo de la primera palabra (B_127: La CNMV suspende la cotización de Bankia), mientras que en alemán se mantiene el artículo de la última forma de la composición, y como si la forma estuviera desarrollada (A_146: Im Januar war der IWF noch von 3,5 Prozent Wachstum ausgegangen).

En cuanto a su ortografía, es frecuente que los acrónimos de neologismos se escriban con letra inicial mayúscula en sus comienzos (Brexit) y con el tiempo pasen a escribirse en minúscula (pyme). Si son sustantivos comunes, el desarrollo de la sigla será también en minúscula (IRPF: impuesto sobre la renta de las personas físicas) y, si se trata de un nombre propio, en mayúscula (ONU: Organización de las Naciones Unidas). Ciertos neologismos vienen entrecomillados las primeras veces que aparecen, y con el tiempo van desapareciendo las comillas (B_230: varios analistas del banco analizan el "Brexit", la salida británica de la UE).

Por último, aunque oralmente se puede formar el plural de las siglas terminando en $s$, en la lengua escrita, la norma indica el uso del artículo para evitar una s final (los CDO, Ios bpa, die CoCos, die SIVS). Estas formas alternan con la s final tanto en español como en alemán. Aquí cabe señalar que, en alemán, y a pesar de que sean siglas traducidas, es frecuente usar el plural de la sigla original en inglés (SIVs: Strukturierte Investment Vehikel). Algunos ejemplos de siglas en plural con su número de apariciones en el corpus son: 


\section{TABLA 2}

Abreviaciones terminadas en $\mathrm{s}$

\section{ESPAÑOL}

- $\operatorname{CDO}(1) / \operatorname{CDOs}(1)$ : Collateralized Debt Obligations

- $\operatorname{CoCos}(16) / \operatorname{cocos}(1)$ : Contingent Convertible Bonds

\section{ALEMÁN}

- $\operatorname{BIP}(29) / B I P s(1):$ Bruttoinlandsprodukt

- $\operatorname{CDO}(3) / \operatorname{CDOs}(1)$ : Collateralized Debt Obligations

- $\operatorname{CoCo}(5) / \operatorname{CoCos}(5)$ : Contingent Convertible Bonds

- SIVs (14)/SIV (2): Strukturierte Investment Vehikel

\subsection{Uso de símbolos}

En el lenguaje económico son frecuentes las abreviaturas simbólicas o símbolos alfabetizables, como \% (por ciento) o $€$ (euro). Son abreviaturas que solo tienen valor gráfico y se pronuncian de forma desarrollada y, al igual que los demás mecanismos de abreviación, sirven para aumentar la efectividad y brevedad de un texto. A diferencia de las abreviaturas, los símbolos no llevan punto final y suelen tener carácter internacional $(\mathrm{kg}, \mathrm{cm}, \mathrm{km}, \mathrm{kcal} . .$.$) . Su uso es dife-$ rente en cada lengua en cuanto a frecuencia y contexto. Así, el símbolo de porcentaje no es frecuente en los textos periodísticos alemanes, y no aparece en el subcorpus alemán, mientras que en el español abundan los símbolos de porcentaje (\%), con 1331 ocurrencias; sin embargo, no hay símbolos de euro ( $€$ ) ni de dólar (\$). En ambos subcorpus se utiliza la forma desarrollada: euros (378) y euro (280) en español y Euro (553), cuyo plural coincide con la forma singular, en alemán. Según Březina (2014: 31), los símbolos se reservan en alemán para las tablas y gráficos, mientras que para las noticias o textos escritos se preferiría la forma desarrollada.

\subsection{Clasificación de categorías semánticas}

Algunos autores ya han clasificado las siglas del lenguaje económico en categorías semánticas. Březina (2014) divide estas formas en subgrupos: índices bursátiles y abreviaturas de nombres corporativos. Muchos de los índices bursátiles, al integrar en numerosas ocasiones cifras, se denominan también siglas alfanuméricas, combinaciones de formas abreviadas con cifras y separadas de ellas por espacios o guiones: Ibex-35, S\&P-500-Index, FTSE 100, Euro Stoxx 50. Gómez de Enterría (1992b), por su parte, se refiere a organismos, sociedades bancarias, sociedades bursátiles, comerciales, industriales o índices bursátiles.

Conforme a las siglas y acrónimos encontrados en nuestro corpus de la crisis económica, podemos hacer la siguiente categorización: abreviaciones de organismos e instituciones (cf. tabla 3), abreviaciones de bancos y nombres comerciales (cf. tabla 4), abreviaciones de productos y operaciones financieras (cf. tabla 5), fondos e índices bursátiles (cf. tabla 6) y otros (cf. tabla 7). 
El grupo más numeroso es el de los nombres propios que denominan organismos e instituciones. Según Gómez de Enterría (1992b: 268), se trata de nombres propios que en su estado desarrollado tienen "una considerable extensión y logran, mediante el uso de la sigla, el acortamiento y la precisión semántica que proporcionan a este lenguaje las características de rapidez y eficacia”. Llama la atención la alta frecuencia en español de la forma abreviada CE (Comisión Europea), mientras que en alemán se emplea únicamente Kommission sin ningún tipo de abreviación.

Respecto de la categoría de fondos e índices bursátiles (Exchange-traded fund / ETF), Steinhauer (2000) afirma que la creación del acortamiento Dax (también escrito en mayúsculas inicialmente) no seguía las normas habituales, ya que se usaban las iniciales de las dos palabras (Deutscher Aktienindex) y la última letra de la segunda. Hoy en día se trata, no obstante, de un procedimiento habitual y nos encontramos con índices como Eurex (Europäischer Index), Stoxx (Stocks Index). En el caso del índice español Ibex, la abreviación consiste en las primeras y las últimas dos letras (Iberia Index).

En una última categoría denominada "Otros" hemos incluido el resto de formas truncadas de nuestro corpus. Como hemos mencionado en páginas anteriores, algunas abreviaturas son más frecuentes en la lengua alemana que en la española. Así, destacan Mill. (29 veces) y Mio. (27 veces) para Millionen y 239 ocurrencias para Mrd. (Milliarden), mientras que el corpus español carece de abreviaturas. Otras siglas se refieren a conceptos macroeconómicos, impuestos o países. Brexit y Grexit son neologismos generados como consecuencia de la crisis, pero hay otras formas truncadas también interesantes, como PIIGS y BRIC. La sigla PIIGS, según la base de datos IATE3, es una “[s]igla peyorativa (por su significado en inglés: «cerdos») que designa a Portugal, Italia, Irlanda, Grecia y España, países todos ellos con problemas en sus balanzas de pagos y déficits públicos”. Ejemplos procedentes del corpus son B_77: cartera de activos en los denominados 'PIIGS' (Portugal, Italia, Irlanda, Grecia y España); A_77: Engagement in den vermeintlich kritischen PIIGS-Staaten - also Portugal, Irland, Italien, Griechenland und Spanien).

\section{TABLA 3}

Abreviaciones de organismos e instituciones

\section{ESPAÑOL}

\section{ALEMÁN}

- AFT (1): Agence France Trésor

- Ascri (1): Asociación Española de Entidades de Capital Riesgo

- CE (28): Comisión Europea

- BaFin (5): Bundesanstalt für Finanzdienstleistungsaufsicht

- $\operatorname{BDB}(1) / B d B$ (1): Bundesverband deutscher Banken

3 Disponible en http://iate.europa.eu/FindTermsByLilld.do?lilld=3548524\&langld=es. 
- CEBS (1)/Cebs (1): Committee of European Banking Supervisors

- CECA (3): Confederación Española de Cajas de Ahorro

- CEIM (1): Confederación Empresarial de Madrid

- CEIOPS (1): Committee of European Insurance and Occupational Pensions

- CEOE (1): Confederación Española de Organizaciones Empresariales

- CESR (1): Committee of European Securities Regulators

- CFTC (1): Commodity Futures Trading Commission

- CHA (1): Chunta Aragonesista

- CNMV (9): Comisión Nacional del Mercado de Valores

- Cores (1): Corporación de Reservas Estratégicas

- EBA (7): European Banking Authority

- Ecofin (12): Consejo de Asuntos Económicos y Financieros de la Unión Europea

- ESFS (3): European System of Financial Supervision

- ESRB (2): European Systemic Risk Board

- Eurostat (6): Oficina Europea de Estadística

- FDIC (10): Federal Deposit Insurance Corporation

- Fed (98): Reserva Federal Estadounidense

- FEEF (9): European Financial Stability Facility

- FMI (172): Fondo Monetario Internacional

- FOMC (1): Federal Open Market Committee

- FROB (18)/ Frob (8): Fondo de Reestructuración Ordenada Bancaria

- FSA (3): Financial Services Authority

- ICV (1): Iniciativa Ciudadana Vasca

- IEE (3): Instituto de Estudios Económicos

- IEO (2): Independent Evaluation Office

- IFO (7): Institut für Wirtschaftsforschung

- INE (3): Instituto Nacional de Estadística

- ISM (2): International School of Management

- Istat (10): Istituto Nazionale di Statistica

- MBIA (9): Municipal Bond Insurance Association

- MEDE(7)/ESM(3):MecanismoEuropeodeEstabilidad

- OCDE (2): Organización para la Cooperación y el Desarrollo Económico

- ONS (1): Office of National Statistics

- OPEP (3): Organización de Países Exportadores de Petróleo

- PP (3): Partido Popular
- BDI (3): Bundesverband der deutschen Industrie

- BIB (1): Bundesinstitut für Bevölkerungsforschung

- BVR (3): Bundesverband der Deutschen Volksbanken und Raiffeisenbanken

- CDU (21): Christlich Demokratische Union Deutschlands

- CEBS (1): Committee of European Banking Supervisors

- CNMV (1): Comisión Nacional del Mercado de Valores

- CSU (2): Christlich-Soziale Union in Bayern e. V.

- DIW (3): Deutsches Institut für Wirtschaftsforschung

- DoJ (2): Department of Justice

- DSGV (1): Deutscher Sparkassen- und Giroverband

- EBA (4)/Eba (2): Europäische Bankenaufsichtsbehörde

- EFAMA (1): European Fund and Asset Management Association

- ESMA (1): European Securities and Markets Authority

- ESRB (2): European Systemic Risk Board

- EU (95): Europäische Union

- EuGH (10): Europäischer Gerichtshof

- Eurostat (5): Europäische Statistikbehörde

- FDIC (9): Federal Deposit Insurance Corporation

- FDP (7): Freie Demokratische Partei

- Fed (70)/FED (4): Federal Reserve

- FROB (10)/ Frob (1): Fondo de Reestructuración Ordenada Bancaria

- FSA (3): Financial Services Authority

- ICO (1): Instituto de Crédito Oficial

- IEO (1): Independent Evaluation Office

- IMK (2): Institut für Makroökonomie und Konjunkturforschung

- Istat (2): Istituto Nazionale di Statistica

- IWF (124): Internationaler Währungsfonds

- MBIA (8): Municipal Bond Insurance Association

- OECD (2): Organisation for Economic Co-operation and Development

- RTC (1): Resolution Trust Corporation

- S \& P (29): Standard \& Poor's

- SaS (3): Sloboda a Solidarita

- SEC (8): Securities and Exchange Commission 
- SoFFin (2): Sonderfonds Finanzmarktstabilisierung (Fondo especial para la estabilización de los mercados europeos)

- S \& P (48): Standard \& Poor's

- UE (86): Unión Europea

- ZEW (3)/ Zew (1): Zentrum für Europäische Wirtschaftsforschung (Centro para la Investigación Económica Europea)

\section{TABLA 4}

Abreviaciones de bancos y nombres comerciales

\section{ESPAÑOL}

- BAT (1): British American Tobacco

- BBK (3): Bilbao Bizkaia Kutxa

- BBVA (46): Banco Bilbao Vizcaya Argentaria

- BCE (211): Banco Central Europeo

- BCP (2): Banco Comercial portugués

- BEI (9): Banco Europeo de Inversiones (European Investment Bank)

- BFA (19): Banco Financiero y de Ahorros

- BNP (12): Banque Nationale de Paris

- BoC (4): Bank of Cyprus

- BOJ (1): Bank of Japan

- Buba (4): Bundesbank

- CAM (3): Caja de Ahorros del Mediterráneo

- Citi (25): Citibank

- DEA (1): Drug Enforcement Administration

- ERT (9): Ellinikí Radiofonía Tileórasi

- FCC (6): Fomento de Construcciones y Contratas, S.A.

- $\operatorname{HBOS}(1)$ : Halifax Bank of Scotland

- HSBC (19): The Hong Kong and Shanghai Banking Corporation

- IKB (7): Industriekreditbank

- KfW (4): Kreditanstalt für Wiederaufbau

- LBBW (2): Landesbank Baden-Württemberg

- MPS (3): Banco Monte dei Paschi di Siena

- OHL (3): Obrascón Huarte Lain

- RBC (2): Royal Bank of Canada

- RBS (39): Royal Bank of Scotland

- WTI (2): West Texas Intermediate
- SIFI (2): Systemically Important Financial Institutions

- SPD (3): Sozialdemokratische Partei Deutschlands

- WTO (1): World Trade Organization

- ZEW (1): Zentrum für Europäische Wirtschaftsforschung 


\section{TABLA 5}

Abreviaciones de productos y operaciones financieras

\begin{tabular}{|c|c|}
\hline ESPAÑOL & ALEMÁN \\
\hline 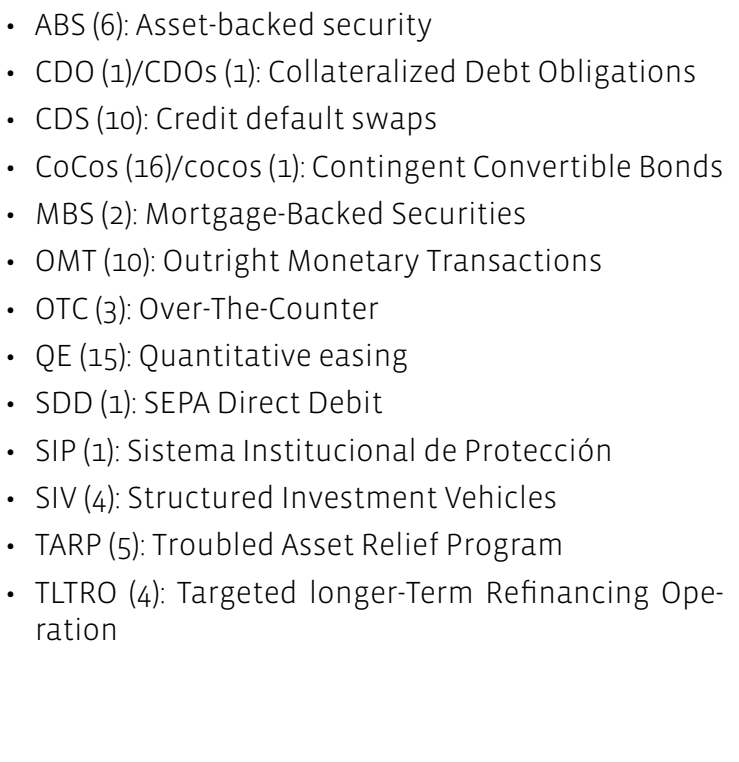 & 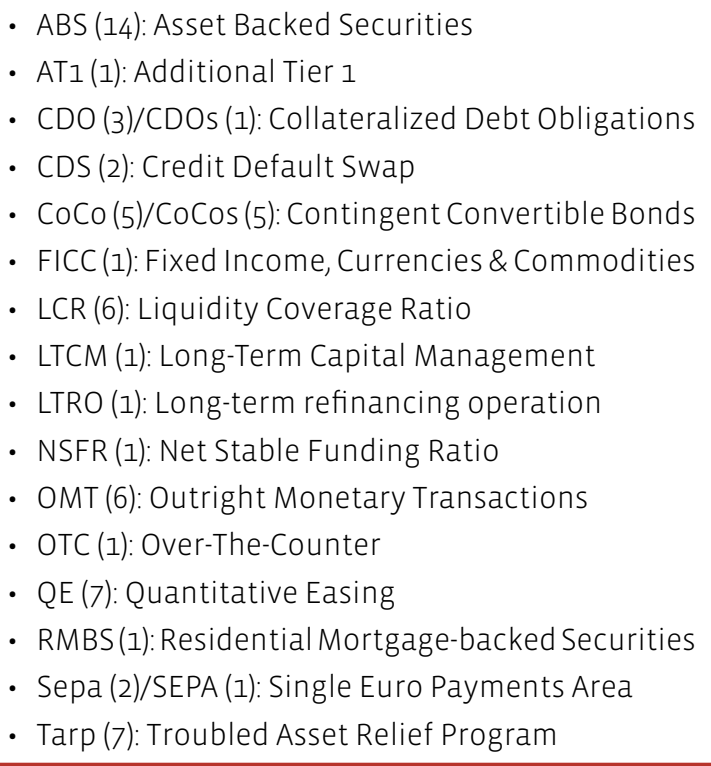 \\
\hline
\end{tabular}

\section{TABLA 6}

Fondos e índices bursátiles

\section{ESPAÑOL ALEMÁN}

- BME (6): Bolsas y Mercados Españoles

- Cac (12): Cotation Assistée en Continu

- Dax (12): Deutscher Aktien Index

- EONIA (1)/ Eonia (1): Euro OverNight Index Average

- ETF (4): Exchange-traded Fund

- Euribor (1): Euro Interbank Offered Rate

- FROB (18)/Frob (8): Fondo de Reestructuración Ordenada Bancaria

- FTSE (1)/Ftse (6): Financial Times Stock Exchange

- IBC (1): Índice Bursátil de Capitalización

- Ibex(64)/IBEX(3): Indice Bursátil de la Bolsa Española

- Nasdaq (25): National Association of Securities Dealers Automated Quotation

- NYMEX (1): New York Mercantile Exchange

- Nyse (2)/NYSE (1): New York Stock Exchange

- PMI (g): Purchasing Managers Index

- PSI (1)/PSI-20 (1): Portuguese Stock Index

- SoFFin (2): Sonderfonds Finanzmarkt-stabilisierung
- Dax (53)/DAX (1): Deutscher Aktienindex

- Eonia (1): Euro OverNight Index Average

- Euribor (8): Euro Interbank Offered Rate

- FROB (11): Fondo de Reestructuración Ordenada Bancaria

- Ibex (4): Índice Bursátil de la Bolsa Española

- L-MDax (1)/L-Dax (1): Late Index

- $\operatorname{MDax}(11) / \operatorname{MDAX}(1)$ : Mid-Cap-DAX

- PSI (1)/PSI-20 (1): Portuguese Stock Index

- TecDax (3)/Tecdax (1): Technologiewerte Index 


\section{TABLA 7}

Categoría "Otros"

\section{ESPAÑOL}

- bpa (4): Beneficio por acción

- Brexit (3): Britain y exit

- CCAA (3): Comunidades Autónomas

- Co. (2)/Co (2): Company

- Grexit (7): Greece y exit

- IRPF(7):Impuestosobrelarentadelaspersonasfísicas

- MEDE (6)/ESM (3)/Mede (1): Mecanismo Europeo de Estabilidad

- MUR (3): Mecanismo Único de Resolución

- MUS (8): Mecanismo Único de Supervisión

- Opa (3): Oferta pública de adquisición

- PEC (2): Pacto de Estabilidad y Crecimiento

- PIB (102): Producto Interior Bruto

- PIIGS (2): Portugal, Italy, Ireland, Greece and Spain

- PPI (2): Payment protection insurance

- pymes(27)/Pymes(1): Pequeñasy medianas empresas

- RETA (1): Régimen Especial de Trabajadores AutónoMOS

- WEO (1): World Economic Outlook

\section{ALEMÁN}

- BIP (29)/BIPs (1): Bruttoinlandsprodukt

- CEO (2): Chief Executive Officer

- Co. (8)/Co (6): Kompanie

- COO (2): Chief Operating Officer

- EFSF (43)/ESM (20)/IFR (1): European Financial Stability Facility

- Grexit (6): Griechenland und Exit

- LCR (6): Liquidity Coverage Ratio

- Libor (8): London Interbank Offered Rate

- LTCM (1): Long-Term Capital Management

- MESZ (2): Mitteleuropäische Sommerzeit

- Mill. (29): Millionen

- Mio. (27)/Mio (1): Millionen

- Mrd. (235)/Mrd (4): Miliarden

- OIBD (1): Operating Income Before Depreciation

- OMT (6): Outright Monetary Transactions

- PIIGS-Staaten (1): Portugal, Italien, Irland, Griechenland und Spanien

- SEPA (1)/Sepa (2): Single Euro Payments Area

\section{Conclusiones}

En este trabajo hemos rescatado estudios lingüísticos de corte teórico sobre el alemán y el español por separado, para posteriormente presentar un estudio contrastivo original sobre un fenómeno frecuente pero poco estudiado en contrastividad en nuestro par de lenguas, el de las formas truncadas. Nos hemos centrado en el lenguaje económico y, específicamente, en el género de las noticias periodísticas de la prensa especializada, donde estas formas adquieren una frecuencia de uso particularmente alta. Así, hemos compilado un corpus comparable de 500 textos (250 textos en alemán y otros tantos en español) sobre la crisis económica y hemos extraído una cifra muy elevada de formas abreviadas: 105 siglas y abreviaturas diferentes en alemán (1532 formas en total) y 113 (1475 en total) en el subcorpus español. Los resultados cuantitativos distribuidos por categoría semántica y lenguas son:

\begin{tabular}{lcc} 
& ALEMÁN & ESPAÑOL \\
Organismos e instituciones & 473 & 588 \\
\hline Bancos y nombres comerciales & 414 & 437 \\
\hline Productos y operaciones financieras & 66 & 80 \\
\hline Fondos e índices bursátiles & 137 & 180 \\
\hline Otros & 442 & 190 \\
\hline TOTAL & 1532 & 1475 \\
\hline
\end{tabular}


Se usa, por tanto, casi un cuatro por ciento más de formas abreviadas en alemán que en español, a pesar de que el subcorpus en español contiene diez mil palabras más que el subcorpus alemán. Con referencia a su corpus correspondiente, estas formas representan el 1,08\% para el subcorpus español y un 1,24\% para el subcorpus alemán, es decir, si los dos subcorpus tuvieran idéntico número de palabras, el alemán contendría casi un quince por ciento $(\mathbf{1 4}, 8)$. Por otro lado, el número de formas abreviadas entre categorías semánticas es muy equilibrado entre las lenguas, si bien en una última categoría más general que hemos denominado "otros" (conceptos económicos, impuestos, leyes y nombres y grupos de países) podría haberse dividido y estudiado con mayor profundidad.

Las siglas y abreviaturas, también en este corpus, se introducen para evitar el uso de denominaciones demasiado largas y lograr un lenguaje preciso, rápido y eficaz. Sin embargo, del estudio también se desprende que las siglas contribuyen a condensar el lenguaje especializado y presentan problemas de traducción que precisan de procesos de documentación intensivos y que pueden ser resueltos con las estrategias expuestas. En este trabajo abordamos la traducción alemán-español de las formas truncadas con ayuda del corpus comparable compilado. Las siglas encontradas en él son, en un alto porcentaje, neologismos procedentes del inglés, por lo que el traductor debe lidiar con una tercera lengua en la traducción alemán<>español. En este punto resultaría útil profundizar en los trabajos de didáctica de la traducción de siglas inglés-español (Rodríguez González, 1990) o inglés-alemán (Göpferich, 2007), que podrían ser útiles desde un punto de vista pedagógico para el traductor alemán-español. Finalmente, en un último punto de este trabajo, hemos elaborado unas tablas en forma de tesauro, es decir, según las categorías semánticas encontradas entre las formas truncadas del corpus, cuya publicación aspira a ser útil a investigadores y traductores.

\section{Bibliografía citada}

BŘEzInA, Jaroslav, 2014: "Phänomen Fachsprachen - Börsensprache unter der Lupe" en Paul Gévaudan y otros (eds.): Philologie im Netz, Phin 70, 17-38 [disponible en http://web.fu-berlin. de/phin/phin70/p70t2.htm, fecha de consulta: 12 de junio del 2018].

Corbacho Sanchez, Alfonso, 2003: “Especificaciones léxico-semánticas del lenguaje económico alemán. Un análisis contrastivo”, Estudios Filológicos Alemanes 3, 273-283.

Corbacho SAnchez, Alfonso, 2006: "Matizaciones conceptuales sobre los lenguajes especializados/conceptual notes on specialized languages”, RLA Revista de Lingüística Teórica Aplicada $1 / 44,67-80$.

Corbacho Sánchez, Alfonso, 2007: "Las abreviaciones en el alemán económico-empresarial y su traducción al español”, Tradução \& Comunicação 16, 17-25.

Duden, 2015: Das Fremdwörterbuch, undécima edición, Berlin: Dudenverlag. 
Elias, Yousif, 1997: "Discours économique et sigles" en Miguel Hernando de LaRRAmendi y Gonzalo Fernandez Parrilla (eds.): Pensamiento y circulación de las ideas en el Mediterráneo, Toledo: Universidad de Castilla La Mancha, 233-246.

Gallardo San Salvador, Natividad, y M. Josefa Gómez de Enterría, 2009: Estudios de léxico especializado, Granada: Traducción en el Atrio.

Gallego-Hernandez, Daniel, 2012: Traducción económica y corpus: del concepto a la concordancia. Aplicación al francés y al español, Alicante: Publicaciones de la Universidad de Alicante.

Gallego-Hernández, Daniel, 2017: "Testing a methodological framework for retrieving parallel texts in the domain of business translation”, Perspectives 25, 1-20.

Gómez de Enterría, M. Josefa, 1992a: "Neología y préstamo en el vocabulario de la economía”, Anuario de Estudios Filológicos 15, 97-106.

Gómez de Enterría, M. Josefa, 1992b: “Las siglas en el lenguaje de la economía”, Revista de Filología Románica 9, 267-274.

Gómez Moreno, Juan Demetrio, 1995: "Las siglas o acronyms, ¿un escollo insalvable en la traducción del business English?” en V Encuentros Complutenses sobre Traducción, actas, Madrid: Universidad Complutense, 575-9.

Göpferich, Susanne, 2007: "Kürze als Prinzip fachsprachlicher Kommunikation" en Jochen A. BÄr, Thorsten Roelcke y Anja SteInHAuer (eds.): Sprachliche Kürze. Konzeptuelle, strukturelle und pragmatische Aspekte, Berlin: De Gruyter, 412-433.

GUILBERT, Louis, 1975: La créativité léxicale, Paris: Librairie Larousse.

Hundt, Markus, y Dorota BiAdalA, 2015: Handbuch Sprache in der Wirtschaft, Berlin/Boston: De Gruyter.

Mateo Martínez, José, 2007: “El lenguaje de las ciencias económicas” en Enrique Alcaraz Varó y otros (eds.): Las lenguas profesionales y académicas, Barcelona: Ariel, 191-203.

Medina Reguera, Ana, y Alice Stender, 2012: "El lenguaje económico español y alemán a través de la prensa especializada. Parámetros para un estudio contrastivo de corpus" en Pilar MARTino Alba y Christiane Lebsanft (eds.): Telar de traducción especializada, Madrid: Dykinson, 79-89.

Medina Reguera, Ana, y Alice Stender, 2013: "Quantifiers in a Spanish and German comparable corpus: a contrastive study based on specialized economic written on-line media texts", Procedia - Social and Behavioral Sciences 95, 372-381.

Michel, Sascha, 2006: "Kurzwortgebrauch”, Germanistische Mitteilungen 64, 69-83.

Moss, Christoph, 2009: Die Sprache der Wirtschaft, Wiesbaden: Verlag für Sozialwissenschaften. 
Pérez Berenguel, José Francisco, 2003: “Glosario de errores comunes en la traducción económica y financiera" en I AIETI. Actas del I Congreso Internacional de la Asociación Ibérica de Estudios de Traducción e Interpretación, vol. 2, Granada: AlETI, 619-628 [disponible en http://www.aieti. eu/wp-content/uploads/AIETI___JPPB_Glosario.pdf, fecha de consulta: 5 de junio del 2018].

Pizarro Sanchez, Isabel, 2011: Análisis y traducción del texto económico inglés-español, La Coruña: Netbiblo.

Real Academia Española, 2009: Nueva gramática de la lengua española, Madrid: Espasa

Rodriguez González, Feliz, 1990: "La traducción de las siglas inglesas" en Felix Rodriguez González (coord.): Estudios de filología inglesa: Homenaje al doctor Pedro Jesús Marcos Pérez, Universidad de Alicante, 169-181.

Rothstein, Björn, 2010: “Was sind Kurzformen?”, Germanistische Mitteilungen 71, 49-69.

SpILLner, Bernd (ed.), 1992: Wirtschaft und Sprache, Frankfurt am Main: Lang.

Springer Gabler Verlag (eds.), 2013: Gabler Wirtschaftslexikon [disponible en http://wirtschaftslexikon.gabler.de, fecha de consulta: 8 de noviembre del 2017].

Steinhauer, Anja, 2000: Sprachökonomie durch Kurzwörter: Bildung und Verwendung in der Fachkommunikation, Tübingen: Narr.

Stender, Alice, 2016: El lenguaje económico alemán y español de la prensa especializada: análisis basado en un corpus de la crisis económica (CRISCORP). Phd Dissertation, Universidad Pablo de Olavide in Seville.

SuAu Jiménez, Francisca, 2010: La traducción especializada (en inglés y español en géneros de economía y empresa), Madrid: Arco Libros. 